\title{
L'aménagement hydroélectrique de Sounda sur le fleuve Kouilou-Niari au Moyen-Congo
}

\author{
The Sounda hydro-electric development \\ on the Kouilou-Niari in the Middle Congo
}

\author{
PAR J. FRANCOU
}

INGENIEUR A ÉLECTRICITE DE FIANCE,

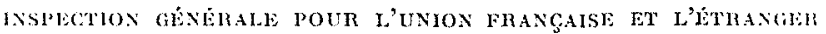

\begin{abstract}
Intiressantes, en grande parlie du fait de len proximité relative $(90 \mathrm{~km})$ da port de Pointe roire, en bordure duquel les industries utilisutrices peuvent s'installer, les ressources hydro-électriques susceptibles d'être aménagée. dans des conditions particulierement anantageuses sur le cours inférieur du fleuve houilonViari au Moyen-Congo, ont fait l'objet d'une itude approfondie, conduite par E.D.F., et apparaissent maintenant comme considérables. L'avant-projet d'aménagement de Sounda com porte, a coté d'ouvrages ou de disposition. classiques (nonte mince en béton de $125 \mathrm{~m}$ de hantenr, évacuatent en saut de ski, usine an pied du barrage), cerlaines particularités dues it la nécessité de concilier au mienx. l'étroitesse de la gorge et l'importance du débit régularisé (1000 $\left.\mathrm{m}^{3} / \mathrm{s}\right)$, notamment la méthode de coupure du fleuve, la conception et l'implantation des prises d'eau, la puissance unitaire des groupes et la disposition de ces groupes dans l'usine, qui méritent d'être signalées.
\end{abstract}

De considérables ressources en énergie hydraulique sont susceptibles d'être mises en œuvre dans des conditions particulièrement avantageuses sur le cours inférieur du fleuve Konilou-Niari.

Les plus importantes d'entre elles sont localisées dans la gorge de Sounda, séparée du port de Pointe-Noire par une distance de $90 \mathrm{~km}$ seulement.

Le parti à tirer des possibilités d'aménage-

\begin{abstract}
Becanse of their relative proximity $(90 \mathrm{~km})$ to the port of Pointe Noirc, aronnd which consumer industries could be set up, and becanse they can be developed under especially favourable conditions on the lower reaches of the Kouilou-Niari in the Middle Congo, E.D.F. made a detailed investigation of hydro-electric resonrces in that area which are now seen to be of considerable magnitude.

In addition to conventional features such as a 125 metre high thin arch concrete dam, a skiinmp spillway and a power house at the foot of the dam, the preliminary design for the denelopment incorporates certain special features which result from the necessity of reconciling the narrowness of the gorge with the large discharge that has to be harnessed $\left(1000 \mathrm{mi}^{3} / \mathrm{s}\right)$. The main such features worthy of being menlioned are the method used to dam the river initially, the design and location of the intakes, the output of each generator and the arrangement of these generator units in the power house.
\end{abstract}

ment offertes par cette gorge paraissant de nature à justifier l'installation d'industries électro-chimiques et électro-métallurgiques à PointeNoire, l'Administration française a fait procéder par Electricité de France à l'étude complète de l'équipement du site de Sounda.

Commencée en juillet 1952 et menée de pair avec celle de l'ensemble industriel et portuaire, cette étude vient d'aboutir à un avant-projet détaillé. 


\section{1. - DONNÊES GẼNẼRALES}

\section{Hydrologie.}

Le bassin versant du fleuve Kouilou-Niari s'etend sur $60000 \mathrm{~km}^{2}$. Situé entre les parallèles 2 et 5 de latitude sud, il appartient a la zone des « climats équatoriaux de transition », lesquels sont caractérisés par l'alternance de deux saisons de pluie (novembre/janvier, avril/juin) et de deux saisons sèehes (lévrier/mars, juillet/ octobre), les unes et les autres de ces saisons clant d'inégale importance.

la pricipitation moyenne interannuelle rayportíe à l'ensemble du bassin versant ressort à $1500 \mathrm{~mm}$ environ. L'humidité relative toujours irès élcvéc of la nébulosité quasi constante limilent à moins de $1200 \mathrm{~mm}$ par an l'évaporation mesurable sur un plan d'eau de grande étendue.

A Sounda, pour une surface drainée de 55000 km², le débit moyen interannuel s'etablit entre
900 ef $1000 \mathrm{n}: \% / \mathrm{s}$. Les fluctuations saisonnieres se situent entre un étiage de $250 \mathrm{~mm}^{3} / \mathrm{s}$ (mi-octohre) et une crue de 3000 ì $4000 \mathrm{~mm} / \mathrm{s}$ (décembre ou avil).

\section{Géologie.}

Avant de se jeter dans l'océan Atlantique, le Kouilou-Niari traverse un petit massif montagneux cotier, le Mayombé. Il coupe une série de chaiuons parallèles orientés du sud-est vers le nord-ouest. Sa vallée présente en conséquence des rétrécissements successifs dont le plus speclaculaire est précisément la gorge de Sounda (ig. 2).

Cette gorge entaille sur $1800 \mathrm{~m}$ de longueur et sur $300 \mathrm{~m}$ de profondeur une crète culminant à l'altitude 400 environ. Elle résulte de l'usure pro-

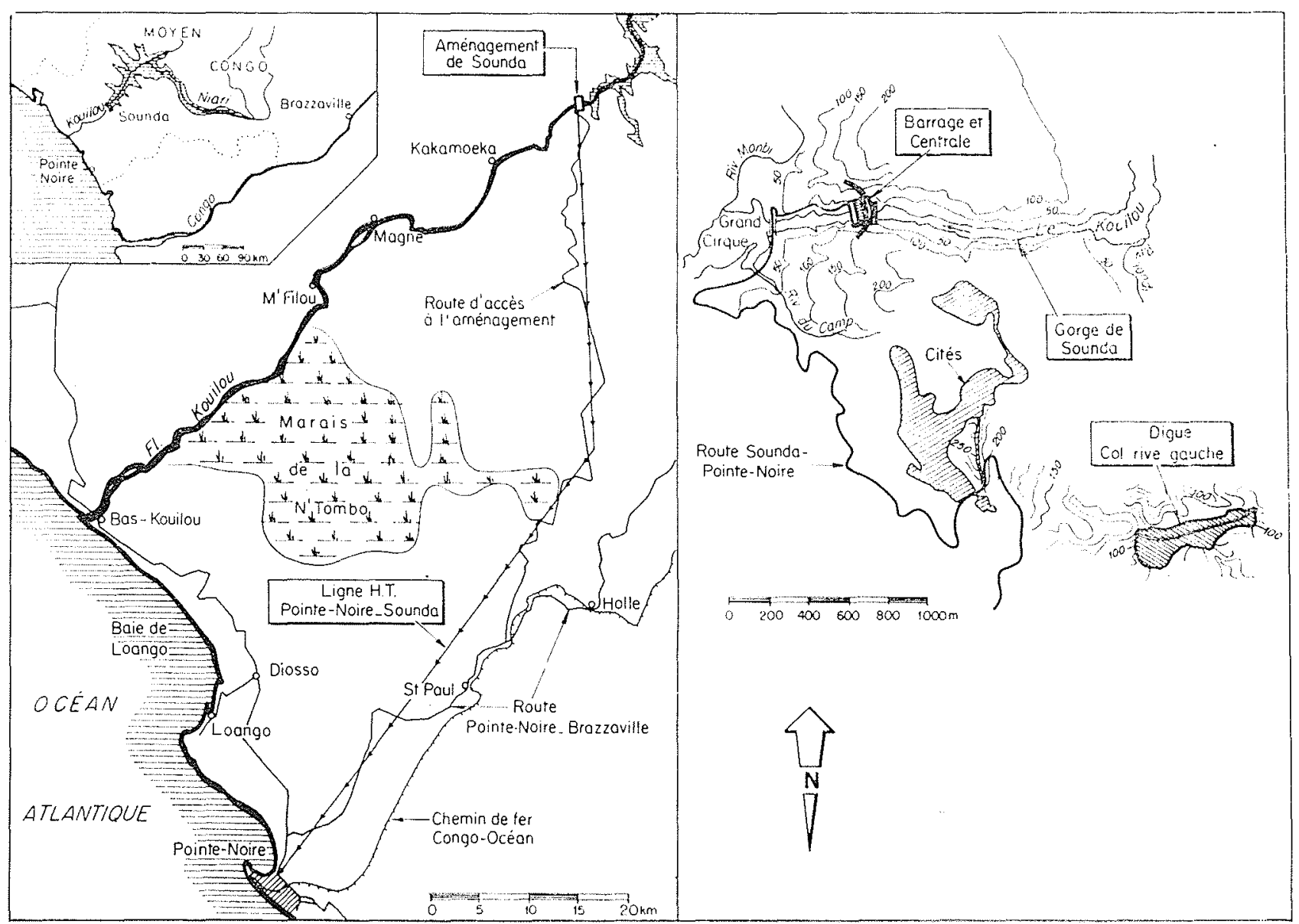

Fic. 1

Carte de situation. 


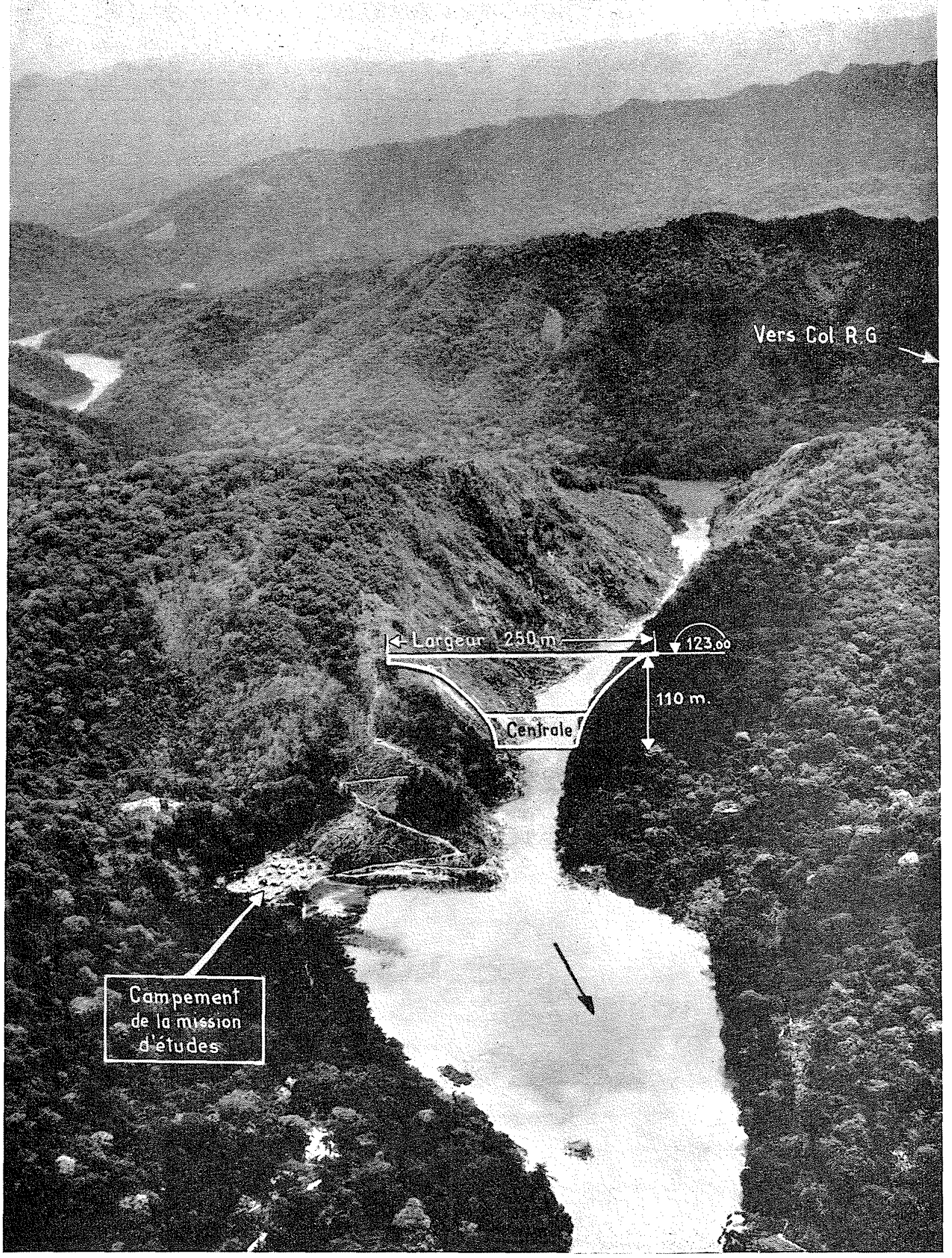

Fig. 2

Photographie de la gorge prise d'avion, en direction de l'amont. 
gressive par le fleuve d'un massif de roches dures (quartzites et gneiss), disposé en couches régulières, et bordé, à l'entrée comme à la sortie du défilé, par aes formations schisteuses beaucoup plus tendres.

\section{Puissance et production de l'aménagement.}

La présence d'un col situé en rive gauche à l'amont de la gorge, et certaines conséquences des submersions ne permettent pas de donner au barrage toute la hauteur quauraient permis de lui accorder l'étroitesse et la profondeur de la gorge, ainsi que la bonne qualité du rocher de fondation.

Cette hauteur a été limitée de telle façon que le niveau normal de retenue s'ètablisse à l'altitude
116,50. A cette altitude, le lac créé par le barrage s'étendra sur une surface de $1600 \mathrm{~km}^{2}$ et se développera sur une longueur de $270 \mathrm{~km}$.

La capacité considérable de ce lac, 35 milliards de $\mathrm{m}^{*}$, permettra d'obtenir une régularisation très poussée des apports, et partant de turbiner à longueur d'années un débit quasi constant approximativement égal au module interannuel du nleuve.

C'est pourquoi les prévisions de production prennent en compte un débit turbinable permanent de $900 \mathrm{~m}^{3} / \mathrm{s}$ auquel correspond une hauteur de chute nette moyenne de $101.50 \mathrm{~m}$. La puissance disponible aux bornes $63 \mathrm{kV}$ du poste abaisseur de Pointe-Noire ressort ainsi à 800000 $\mathrm{kW}$ et la production annuelle d'énergie livrable aux consommateurs, à 7 milliards de $k W h$.

\section{II. - DESCRIPTION DE L'AMENAGEMENT. - GÉNIE CIVIL}

\section{Disposition générale des ouvrages.}

De toutes les dispositions d'ouvrages que la topographie du site permet de concevoir, celles comportant une usine extérieure implantée juste au piea du barrage se sont révélées en fin de compte comme les plus avantageuses.

En effet, loutes les autres solutions: usines souterraines, usines installées au débouché aval de la gorge, exigent un système hydraulique relativement développé dont le projet et la réalisation soulevent des difficultés techniques qu'aggravent l'importance même du débit équipé (1 $200 \mathrm{~m}^{3} / \mathrm{s}$ au stade final) et les conditions de stabilité de marche des groupes en réseau séparé.

Bien entendu, l'implantation de l'usine à l'aval immédiat du barrage pose, elle aussi, certains problèmes. Le plus important est celui qu'entraine l'exiguité de la place disponible dans le sens transversal, à l'emplacement choisi.

Il a été possible de s'accommoder sans trop de difficulté d'une telle sujétion, grâce à l'adoption de puissances unitaires élevées, et à la disposition des groupes «en quinconce» sur deux axes parallèles. Il y sera revenu plus loin.

Cependant, le fractionnement de la puissance installée n'est pas fixé de façon définitive. Deux variantes sont encore en présence, qui s'accommodent de la même disposition générale des ouvrages et comportent :

- la première, six groupes de $218 \mathrm{MW}$,

- la deuxième, huit groupes de $156 \mathrm{MW}$,

l'un quelconque de ces groupes étant considéré, dans l'un et l'autre cas, comme une unité de réserve.

Il convient aussi de préciser que dans chacune des hypothèses de fractionnement considé- rées, l'installation du dernier groupe n'interviendra qu'après la dérivation, dans le bassin du Kouilou, du cours supérieur d'un fleuve voisin : la Nyanga. Cette dérivation, qui n'a pas encore été étudiée d'une facon détaillée, f'era l'objet d'une phase ultérieure de travaux. Sa productibilité viendra s'ajouter à celle qui vient d'ètre indiquée tout à l'heure, et qui s'entend done pour le Kouilou seul, équipé de $(n-1)$ groupes.

La description donnée ci-après correspond à la première hypothèse de fractionnement : usine à 6 groupes (fig. 3).

Les principaux ouvrages de génie civil : barrage, évacuateurs, prises d'eau, usine... sont rassemblés dans la première moitié du bief aval de la gorge. Leur exécution est subordonnée à celle de deux galeries de dérivation et de deux batardeaux.

Le seul ouvrage qui se trouve à l'écart du chantier principal est la digue à construire sur le col rive gauche pour l'exhausser de l'altitude 102 à l'altitude 125 .

\section{Ouvrages provisoires.}

Les problèmes que posent la coupure du courant et la réalisation des batardeaux sont assez particuliers. La difficulté de ces deux opérations résulte non pas tellement de la violence du courant ou du volume des travaux, mais plutòt du fait qu'il faut obligatoirement les me. ner jusqu'à leur terme durant une seule grande saison sèche, c'est-à-dire entre un $1^{\text {cr }}$ juin et un 15 octobre.

Avant de parler de la solution envisagée pour résoudre ces problèmes, il convient de dire quelques mots des galeries de dérivation projetées, qui mesureront l'une $375 \mathrm{~m}$ et l'autre $435 \mathrm{~m}$ de 


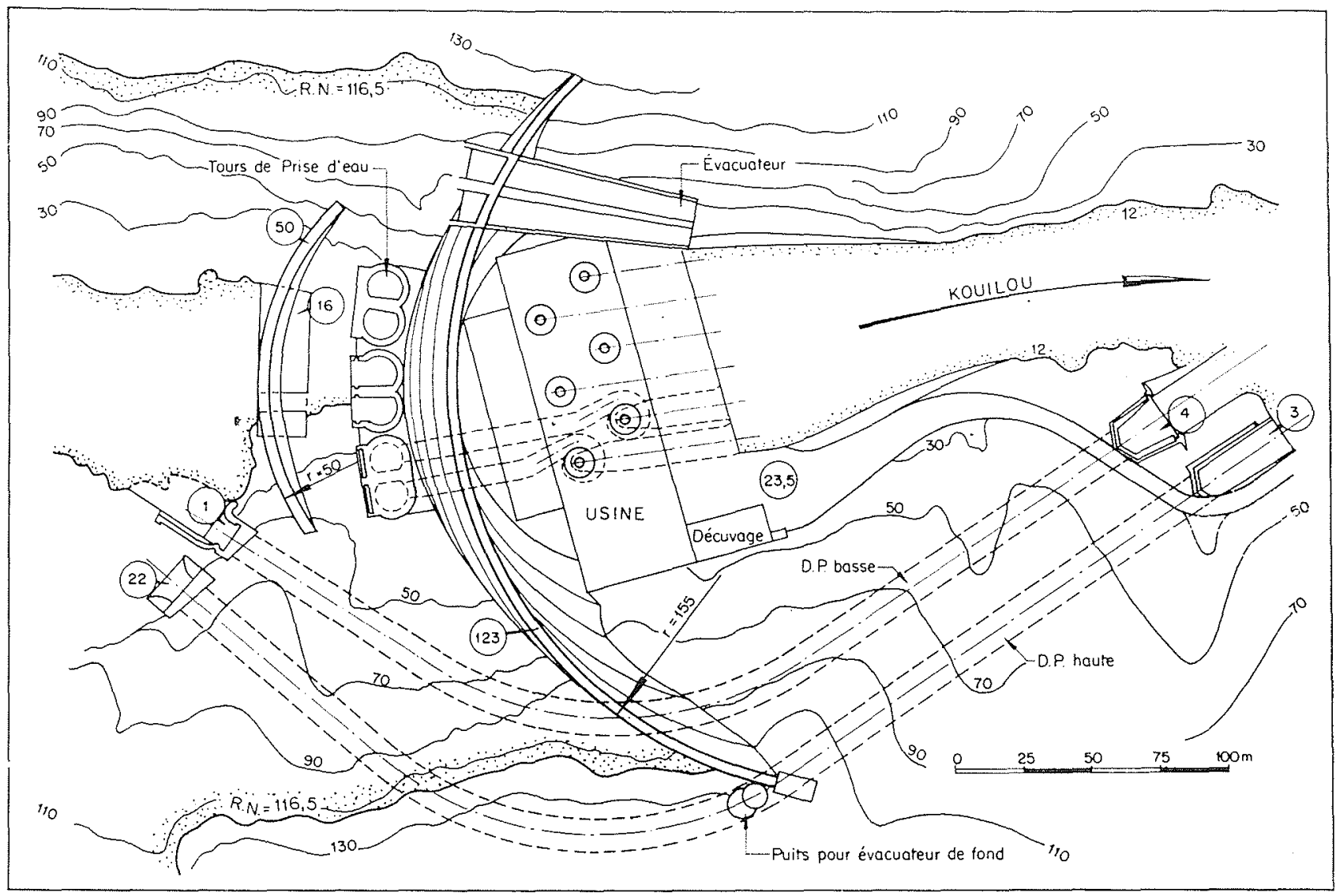

FIG. 3

Plan d'ensemble de f'aménatgement.

Iongueur. Ce sont des ouvrages de section inportante $\left(113 \mathrm{~m}^{2}\right)$; section circulaire, de diamètre intérieur $12 \mathrm{~m}$, un revêtement de béton légerement armé protégeant le rocher contre l'érosion.

I a galerie la plus courte a son seuil d'entrée calé à la cote 1 , et son radier dans la section de sortie à la cote - 2, alors que le niveau du Louilou à l'étiage s'établit aux environs de la cote 9 . Cette galerie a donc été calée très bas de facon que la dénivelée artificielle à créer pour contraindre le fleure a quitter son lit au moment de la coupure ne dépasse pas $2 \mathrm{~m}$ si le dóbit reste inférieur à $600 \mathrm{~m}^{3} / \mathrm{s}$.

La galerie la plus longue a son seuil d'entróe calé à la cote 22; elle entre en service lorsque le débit à dériver devient supérieur à $1500 \mathrm{~m} / \mathrm{s}$.

Le débit évacué par ces dérivations peut atteindre $4000 \mathrm{~m}^{3} / \mathrm{s}$ : le plan d'eau derriere le batarteau tendrait alors à la cote 48 .

$$
\text { : }
$$

La méthode à suirre pour couper le courant n'est pas encore arrètée définitivement. Tontefois, celle préconisée par M. Coyne et qui consiste à basculer dans le courant un massif de béton de $45 \mathrm{~m}$ de hauteur environ, fait actuellement l'objet d'une étude approfondie.

la figure n" 4 donne une idée de la topographie et de la nature des rives à l'emplacement choisi pour la coupure, qui se trouve ici vu de l'amont. C'est sur la berge rive droite que serait fabriqué le bloc avant de le basculer.

Les dispositions représentées sur la figure 5 correspondent au stade actuel des études et des essais; leur mise au point définitive exigera cerlaines modifications, qui n'altéreront pas cependant le principe de l'opération.

On cherche d'une part à obturer presque complitement le lit du fleuve, et d'autre part à conslituer un massif de fondation sur lequel la voùtelatardeau viendra s'inscrire dans sa partie centrale.

Ceci conduit aux dimensions suivantes : hauteur $45 \mathrm{~m}$, épaisseur (dans le sens amont aval) $18 \mathrm{~m}$, plus grande largeur $21 \mathrm{~m}$. Le volume de bóton à mettre en ouve atteint $11000 \mathrm{~m}^{3}$, socle non compris, et la masse à baseuler, quelque 28000 tonnes. 
Fin. 4

limplacement de la coupure rue d'avion, en direction de l'aval.

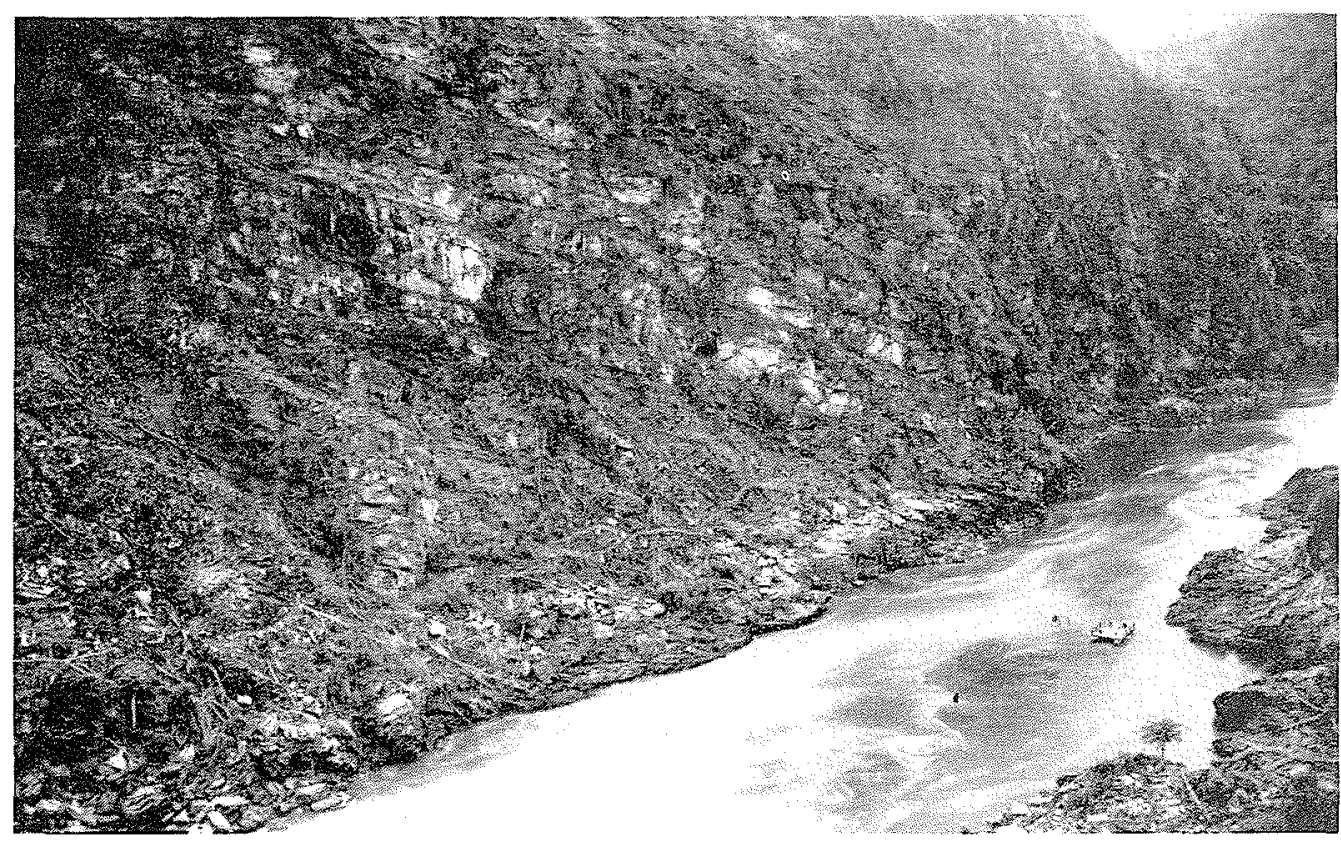

Fli, ;

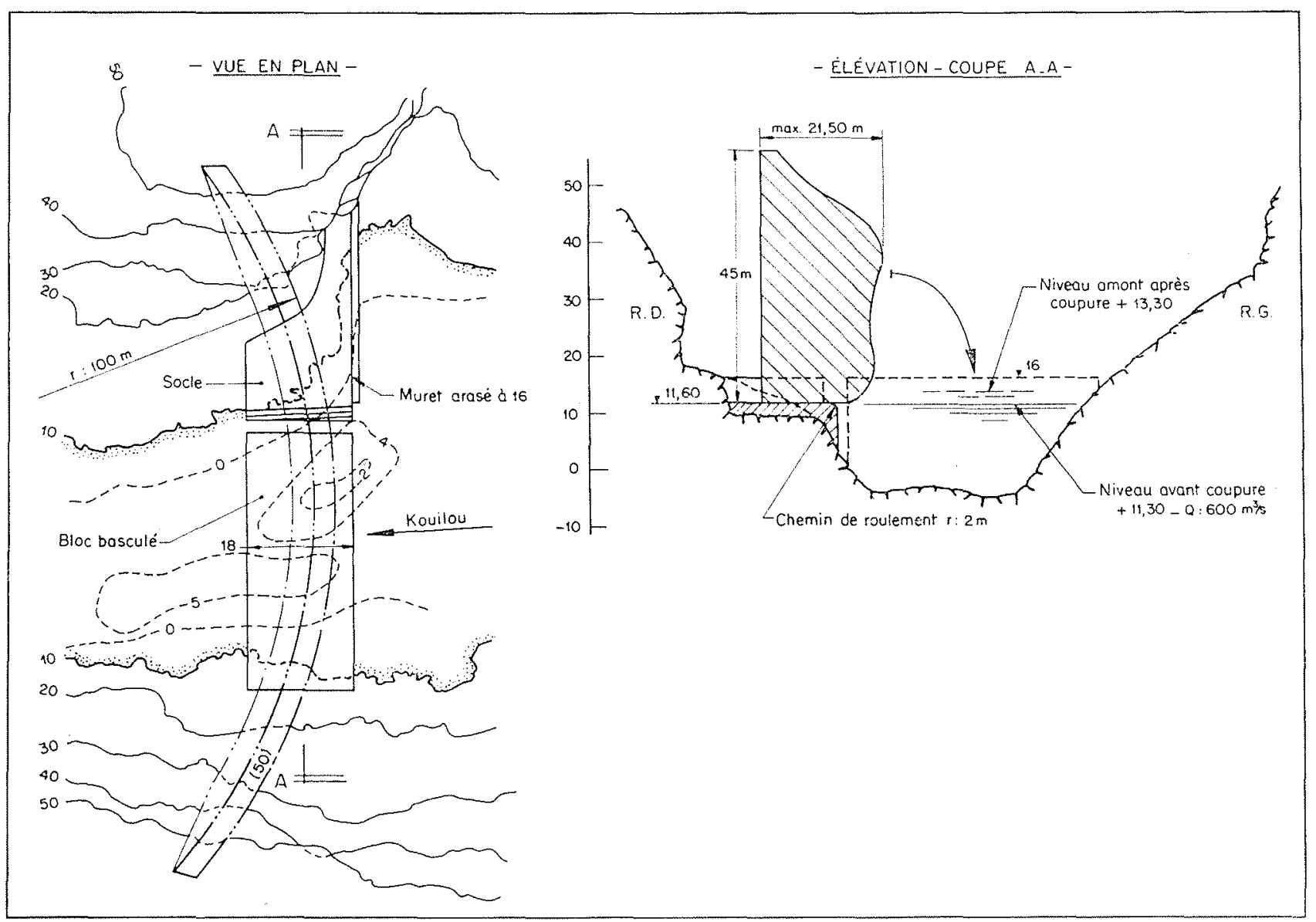


Le Laboratoire d'Hydraulique de Chatou a réalisé un modèle au $1 / 75^{\circ}$ à l'aide duquel ont été déterminées :

1. l'implantation, la cote d'arase et la forme du socle sur lequel le bloc sera édifié;

$2^{\circ}$ la position idéale de ce bloc, l'objectif étant d'obtenir :

- d'une part une chute et une position finale aussi réitérables que possible,

- d'autre part, une trajectoire telle qu'il ne se produise pas d'impact localisé prématuré susceptible de briser le bloc avant sa mise en place.

L'étude cinématique a été faite à l'aide d'une caméra permettant de prendre 2000 images par seconde. Les résultats suivants tendent à se dégager des essais effectués jusqu'à ce jour :

- Le bloc tombe à coup sûr de la façon prévue à l'emplacement choisi pour une position de départ bien déterminée sur son socle et pour une forme particulière du « chemin de roulement » sur lequel sa face d'appui pivote et glisse pendant le basculement. Cette forme est donnée par un arrondi de rayon 2 mètres dont la génératrice est légèrement biaise par rapport à l'arête de la face d'appui;

- La rugosité plus ou moins grande du chemin de roulement n'exerce aucune influence notable sur les trajectoires;

- Si le socle est arasé à la cote 11,60, la face supérieure du bloc basculé passe par la position horizontale au moment même où la chute s'achève.

Ce dernier résultat présente un grand intérêt : en effet, si une telle coöncidence est réalisée, la face modelée vient épouser directement le lit, au terme d'une trajectoire continue, sans avoir au préalable heurté un point particulier de la berge rive gauche ou du fond.

Les essais vont ètre poursuivis en vue d'apporter au procédé de nouvelles améliorations. On cherchera d'une part à réduire le plus possible la brèche qui subsiste en rive droite après le basculement du bloc, brèche qu'il faudra bétonner à l'abri d'une enceinte de palplanches ou de coffrages glissants.

On s'efforcera d'autre part de retoucher les implantations de façon que toute la surface de fondation du socle soit située au-dessus du niveau d'étiage; si l'on y parvenait, l'exécution de cet ouvrage s'en trouverait considérablement facilitée.

D'autres essais permettront de vérifier si la vitesse du courant est susceptible d'influer sur la trajectoire, ce qui paraît peu probable, et de déterminer comment la force vive du bloc est absorbée, partie par la masse liquide formant matelas amortisseur, partie par la conche d'éboulis de 2 à 4 mètres d'épaisseur qui recouvre le fond rocheux sur les deux tiers de la surface d'impact.

Enfin, le dispositif à utiliser pour amorcer le basculement sera mis au point également sur modèle réduit. Il semble que la meilleure façon de procéder à cet égard consiste à construire le bloc en maintenant une faible prépondérance du moment stabilisateur sur le moment de renversement. A l'instant choisi on pourra déséquilihrer l'ouvlage soit en lui donnant unc impulsion par l'entremise de vérins par exemple, soit en dynamitant des étais.

$$
\text { * }
$$

Le batardeau amont est une voute en béton; toute autre sorte d'ouvrage se trouve d'ailleurs exclue par la faible distance qui sépare l'entrée des galeries de dérivation du massif des prises d'eau. Le couronnement de cette voûte est arasé à la cote 50. Si la coupure est réalisée comme il vient d'être indiqué, l'ouvrage sera fondé sur le bloc basculé qui émergera à la cote 16 . Si l'on procède d'une autre façon, si l'on choisit par exemple de couper le fleuve en réalisant le batardeau aval, la voute descendra jusqu'au point le plus creux du thalweg, soit à la cote -.- 5 environ.

Le batardeau aval est un massif d'enrochements étanché par un rideau central de palplanches.

\section{Barrage principal.}

L'étroitesse de la vallée et la solidité du rocher sur chaque versant ont permis au Bureau André Coyne et Jean Bellier de porter le choix sur une voûte mince en béton. Pour remédier aux difficultés d'exécution inhérentes à la concentration des principaux ouvrages dans un espace restreint, cette voûte est désolidarisée complètement des prises d'eau, et séparée autant que faire se peut de l'usine.

Dans sa partie centrale, elle repose au niveau 15 sur un socle massif qui enrobe les conduites foreées jusqu'au mur amont de l'usine, et sert de fondation aux tours de prise d'eau. Côté rive droite, elle prend directement appui au rocher; coté rive gauche, l'appui se fait par l'intermédiaire de l'évacuateur de surface.

Arasé au niveau 123, son couronnement laisse subsister au-dessus de la retenue normale (cote 116,50) un creux de $6,5 \mathrm{~m}$ réservé à l'amortissement de la crue exceptionnelle : débit maximum $8000 \mathrm{~m}^{3} / \mathrm{s}$.

Le rayon du cercle d'implantation est de $155 \mathrm{~m}$; les parements de la voûte sont des surfaces à rayons variables, et présentent dans certains profils des surplombs de l'ordre de plu- 
Fici, 6

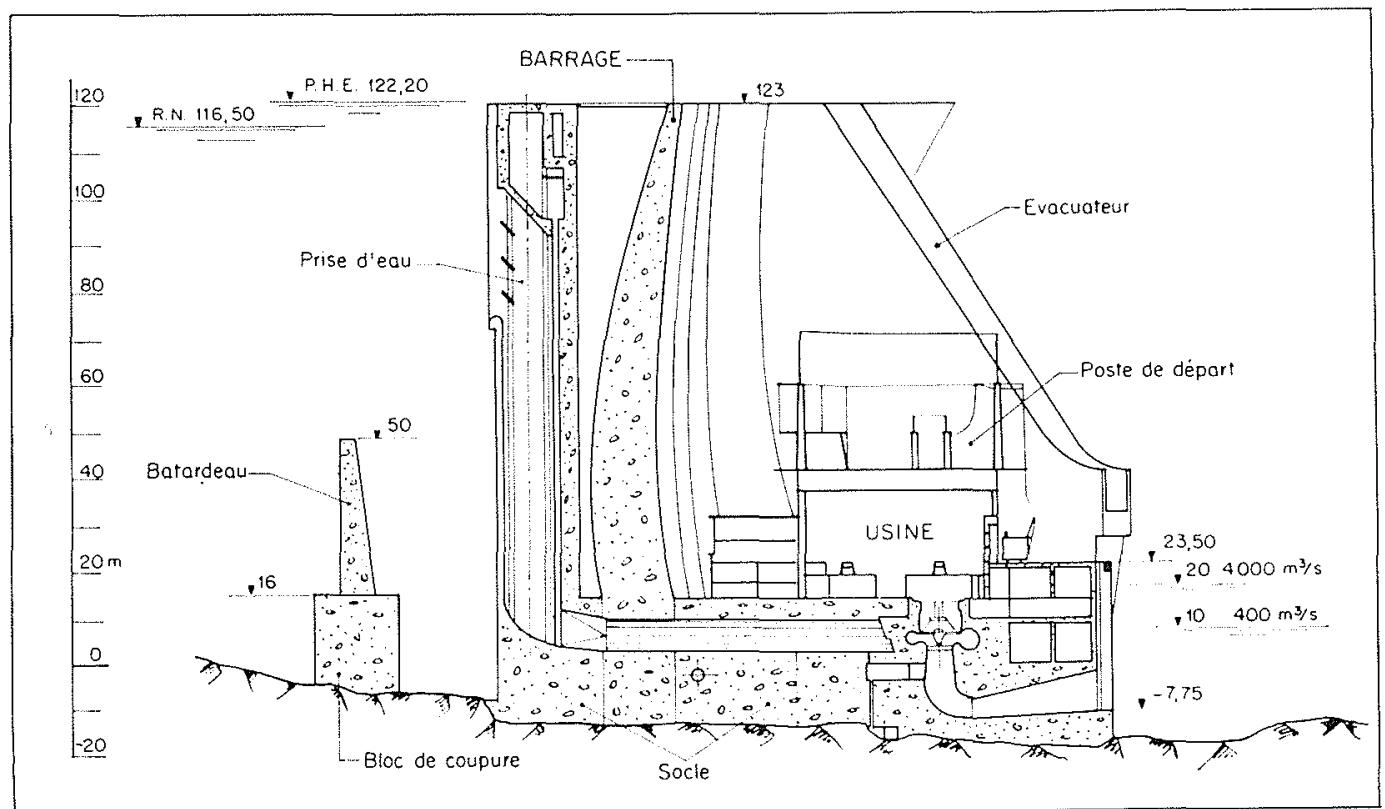

sieurs mètres. Vis-à-vis des proportions générales, les épaisseurs prévues sont relativement faibles : $3,60 \mathrm{~m}$ à la partie supérieure, 13 à $15 \mathrm{~m}$ au $\mathrm{ni}$ veau du socle. Malgré cette finesse, la compression moyenne des arcs reste inférieure à $60 \mathrm{~kg} / \mathrm{cm}^{2}$.

On voit donc qu'il s'agit d'un ouvrage dont la taille, aussi bien que les formes, n'ont rien d'exceptionnel.

\section{Prises d'eau et conduites forcées (fig. 6).}

Les diverses sections du système d'amenée atteignent par contre des dimensions inusitées. Pour une chute de cet ordre, le débit unitaire adopté est exceptionnel puisque, dans la solution décrite, il s'élève à $240 \mathrm{~m}^{3} / \mathrm{s}$, valeur rarement dépassée, si ce n'est pour équiper certaines chutes de faible hauteur au fil de l'eau.

L'impossibilité dans laquelle on se trouvera de vidanger la retenue dans un délai raisonnable conditionne dans une large mesure la disposition de l'ouvrage de prise proprement dit. Pour qu'un accident survenant à une vanne de garde soit rapidement réparable, il faut qu'on puisse obturer facilement le pertuis d'admission; cela conduit à caler le seuil de prise d'eau au plus haut niveau compatible avec une exploitation suffisante de la réserve, de facon que les éléments de batardeau constituant le système de bouchure soient à manœuvrer sous une charge modérée. Par ailleurs, la position élevée des grilles laisse à des « hommes-grenouilles 》 la possibilité d'éliminer des arbres morts flottant entre deux eaux jusqu'à venir s'échouer contre les harreaux.

L'avant-projet des ouvrages d'amenée tient compte de ces sujétions.

Les six prises d'eau reposent sur le socle gé- néral, au pied amont de la voûte. Elles sont constituées essentiellement par des tours cylindriques jumelées de $108 \mathrm{~m}$ de hauteur et de $13 \mathrm{~m}$ de diamètre intérieur. Les pertuis d'admission, dont le seuil est calé au niveau 76,50, échancrent leur face amont sur une largeur de $10 \mathrm{~m}$ et une hauteur de $28 \mathrm{~m}$. Ils sont équipés d'une grille métallique relevable prenant appui sur trois voiles en béton armé.

Logée dans la partie basse de la tour, la vamme de garde de la turbine obture un pertuis rectangulaire de $8 \mathrm{~m}$ de hauteur et $6 \mathrm{~m}$ de largeur. Elle est mancuvrée par un servo-moteur installé dans le puits, au-dessous du niveau 123.

Les conduites forcées sont horizontales : cette disposition permettra d'abréger l'interruption de bétonnage nécessaire à leur mise en place et partant, de "sortir 》 rapidement le socle et la partie basse de la voûte.

Les exigences de la régulation imposent un diamètre intérieur de $7 \mathrm{~m}$, auquel correspond une épaisseur de tôle de l'ordre de $50 \mathrm{~mm}$.

\section{Evacuateurs de crues.}

L'aménagement comporte deux ouvrages d'évacuation :

- un déversoir de surface, en saut de ski, accolé à la falaise sur laquelle la voûte vient prendre appui en rive gauche;

- un évacuateur de fond installé après coup dans la dérivation haute.

Ces deux ouvrages sont de conception classique; leur capacité totale d'évacuation est limitée à $3300 \mathrm{~m}^{3} / \mathrm{s}$, bien que le débit de pointe de la crue exceptionnelle prise en considération atteigne $8000 \mathrm{~m}^{3} / \mathrm{s}$. Une telle réduction est rendue possible par l'amortissement que procure la 


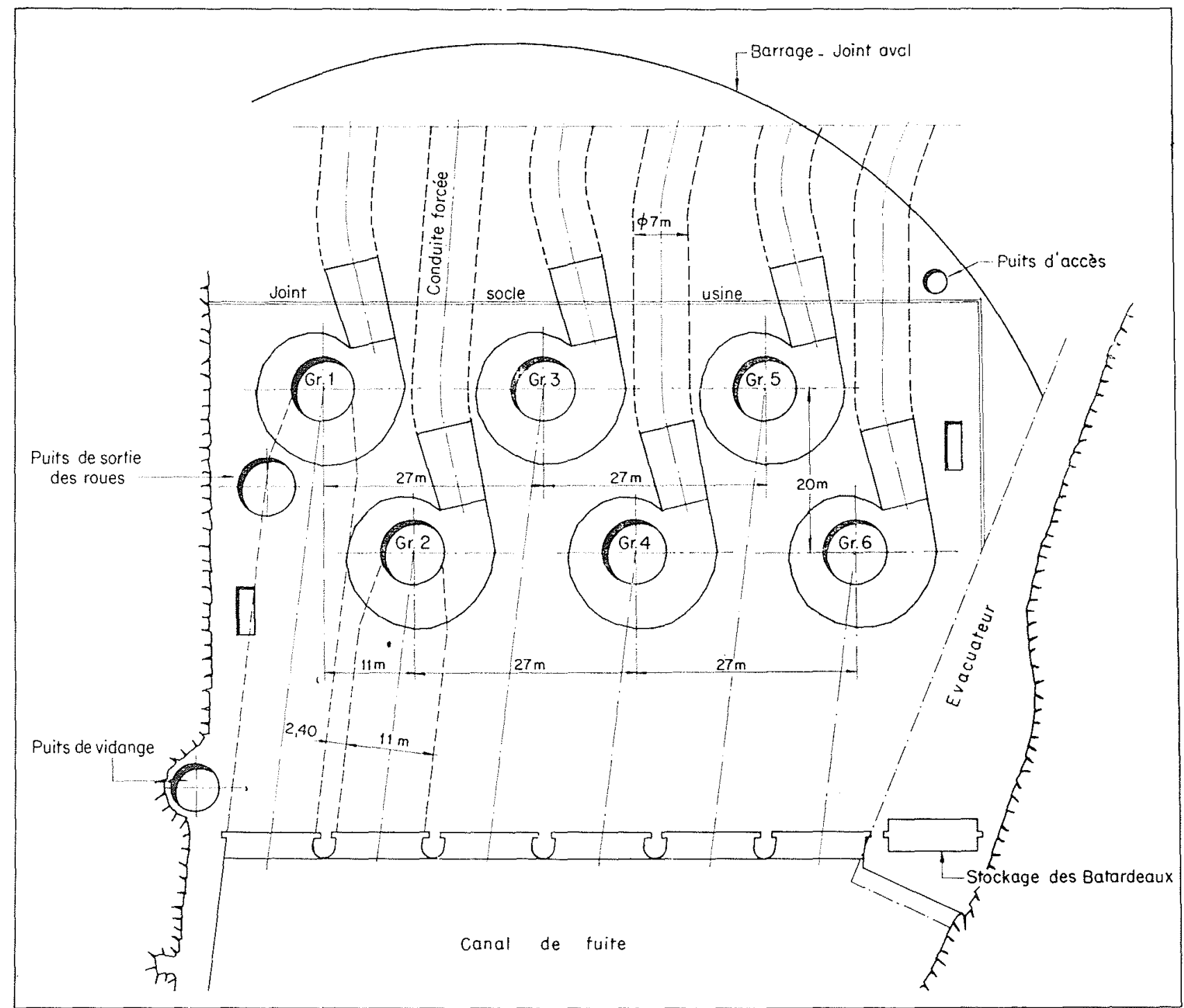

Fig. 7

tranche supérieure de la retenue, entre les niveaux 116,50 et 122,20 .

\section{Usine.}

Comme indiqué tout à l'heure, la possibilité de placer l'usine à l'aval immédiat du barrage résulte de l'adoption de puissances unitaires élevées, et de la disposition des groupes en quinconce sur deux files parallèles.

Une usine rectiligne équipée de 12 groupes de $100 \mathrm{MW}$ alignés sur une seule file aurait une longueur de $250 \mathrm{~m}$; avec 8 groupes de $156 \mathrm{MW}$, elle aurait encore $190 \mathrm{~m}$; avec 6 groupes de $218 \mathrm{MW}$, elle n'en aurait plus que 160 environ. La disposition en quinconce permet encore de gagner sur l'encombrement transversal, puisque la longueur du bâtiment projeté n'est plus que de $118 \mathrm{~m}$ au niveau de la plateforme de montage.

Malgré tout, il faut encore encastrer lusine dans la rive droite, et le déroctage à réaliser s'étend jusqu'à l'aplomb de la courbe de niveau 45 du versant. Cet encastrement reste iniportant, mais n'est plus prohibitif; il serait encore acceptable avec 8 groupes de $156 \mathrm{MWV}$ disposés en quinconce, mais il faudrait alors choisir une implantation en arc de aercle, et reduire considérablement la surface de la plateforme de montage.

La figure 7 montre la disposition des conduites forces, des bàches ef des aspirateurs au niveau le plus encombré de l'infrastructure.

A noter que la disposition en quinconce se traduit par un certain pincement des aspirateurs, qui ont une section de sortie de $8 \mathrm{~m}$ par $11 \mathrm{~m}$ presque aussi haute que large.

D’autre part, les aspirateurs des groupes amont ont une longueur surabondante pour les seuls besoins de la récupération d'énergie. 
On s'est aperçu, en effet, au cours des essais sur modèle réduit, que si l'on voulait éviter d'importantes oscillations du plan d'eau aval, il fal. lait restituer le débit turbiné dans une section transversale du canyon commune à tous les groupes.

Les autres caractéristiques de l'usine n'appellent pas de commentaire particulier. L'infri- structure, jusqu'au niveau de la plateforme de montage (cote 23,50) est réalisée en béton plus ou moins armé; la superstructure, qui s'élève jusqu'au niveau 43,50 , est un bâtiment à ossature métallique dont la toiture-terrasse supporte l'appareillage et les travées haute tension du poste de départ.

\section{III. - GROUPES TURBINES-ALTERNATEURS}

Chaque groupe est autonome : comme on l'a vu tout à l'heure, la prise d'eau et la conduite forcée qui l'alimentent sont indépendantes des autres ouvrages d'amence.

Une consultation très large faite auprès des constructeurs français et étrangers permet de dégager les principales caractéristiques des groupes.

TURBINES :

La turbine est du type Francis a axe vertical.

- Hauleur de chute nette... 101 in

-.. Débit nominal . . . . . . . $240 \mathrm{~m}^{3} / \mathrm{s}$

- Rendement sous $102 \mathrm{ml}$ à $85 \%$ d'ouverture......... $93,2 \%$ $218 \mathrm{MW}$

Puissance nominale....... $125 \mathrm{tr} / \mathrm{mln}$

- Vitesse de rotation. . . . . . . .

Les dimensions el poids des éléments princ1paux, tels qu'ils découlent de la moyenne des renseignements fournis par les différents constructeurs, sont les suivants :

Diamètre maximum de la bâche . . ............

- Diamère de la roue......

- Poids de la rone.........

- Poids de l'arbre..........

-... Charge sur le pirot.......

Alternateurs :

- Puissance nominale.......

-..- Facteur de puissance nominale . . . . . . . . . . . .

- Tension nominale : de l'ordre de ......................

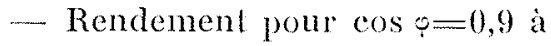
$85 \%$ de charge. . . . . . . .

- Moment d'inertie........ $50000 \mathrm{t} / \mathrm{m}^{2}$

Tels qu'ils découlent de la moyenne des renseignements fournis, les dimensions et poids sont :

- pour le stator :

diamètre extérieur...... $12 \mathrm{~m}$

poids ........... $350 \mathrm{t}$

- pour le rotor :

diamètre.......... $10 \mathrm{~m}$

poids ............660 600

\section{Problèmes de construction des groupes.}

Etant donné les dimensions importantes des groupes, une enquète a cté faite auprès des forges et fonderies pour roir si les moyens dont disposent les métallurgistes permettent l'exéculion de pieces de cette taille.

parallèlement à cette investigation, on s'est assuré chez les usiniers que le parc en machinesoutils permettant d'usiner des pieces de grandes dimensions était également suffisant.

Ces deux enquêtes ont été favorables el aucune impossibilité n'apparaît.

Ces informations se sont trouvées confirmées par les propositions recues des constructeurs, qui n’ont marqué aucune réticence pour la réalisation de groupes de celle importance.

D'autre part, il est à noter que des turbines de mèmes dimensions sont actuellement en cours de construction chez un constructeur nordique, et que, prises l'une après l'autre, des pieces atussi importantes ont dójà ćté exécutées pour divers aménagements.

En ce qui concerne la pivoterie, la charge sur celle-ci est d'environ 1400 tonnes, soit de $10 \%$ supérieure aux charges des pivots des aménagements du Rhin el du Rhône (Fessenheim, Chàteauneuf).

La construction des alternateur's ne pose pas de problème spécial.

Les différentes enquètes auxquelles il a été procédé ont, en outre, montré que la tendance générale était a l'augmentation de la puissance unitaire des groupes, et que des unités hydrauliques de $200 \mathrm{MW}$ en 1964 s'inscrivaient dans l'evolution normale des grands equipements.

\section{Problèmes de transport.}

Les moyens de manutention existant au port de Pointe-Noire permettent de décharger des colis d'un poids maximum de 100 tonnes.

Or, le colis le plus lourd est constitué par la roue $(95 \mathrm{t})$, ce qui dispense de renforcer les installations existantes.

Le transport pai route de pieces de ce poids est devenu classique : malgré l'importance des groupes, le transport du matériel à pied d'œuvre ne pose done pas de problèmes particuliers. 


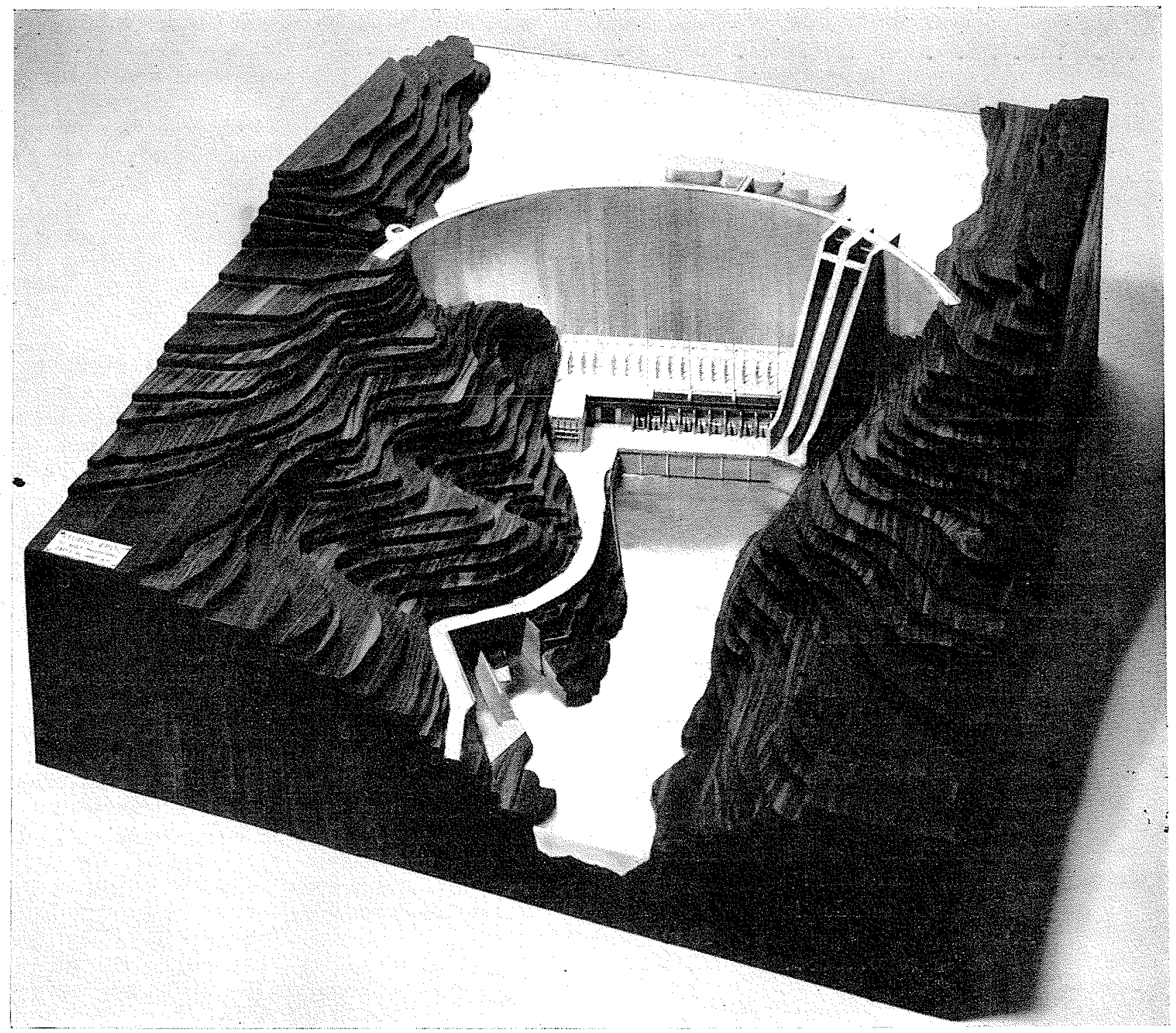

FIG. 8

Maquette (Pavillon de la France a l'Exposition de Bruxelles).

\section{IV. - EVACUATION DE L'ENERGIE}

\section{Transformateurs.}

Plusieurs types d'appareils ont été envisagés en fonction de la tension et du nombre des groupes. A chaque type correspond un schéma électrique différent.

Par exemple un transformateur triphasé de 242 MVA peut être associé en schéma-bloc à chaque alternateur.

\section{Lignes.}

Deux tensions de transport ont été étudiées : $380 \mathrm{kV}$ et $220 \mathrm{kV}$. Cette dernière a finalement été retenue.
Cette tension nécessite la construction de deux lignes, équipées de deux conducteurs $595 \mathrm{~mm}^{2}$ Alu-Acier par terne.

Pour pouvoir transporter toute la puissance, mème en cas d'arrèt d'une des lignes, il faut prévoir une ligne supplémentaire de réserve.

\section{Poste de Pointe-Noire.}

L'énergie serait livrée aux utilisateurs à la tension de $63 \mathrm{kV}$. Une partie importante de l'énergie réactive nécessaire aux industriels scrait fournie sur place par une batterie de condensateurs branchée en $63 \mathrm{kV}$. 


\section{V. - CONCLUSION}

La réalisation de l'aménagement hydro-électrique de Sounda s'étalera sur six ou sept années de travaux dont deux au minimum seront consacrées à la mise au sec des fouilles.

Il y a lieu de tenir compte des difficultés inhérentes à la situation géographique du site, difficultés qui tendent toutes à renchérir le coùt des travaux : éloignement des usines fabriquant le ciment et le matériel d'équipement, absence de main-d'œuvre qualifiée locale, cherté de la maind'ouvre expatriée, etc.

L'entreprise est d'importance, puisqu'il faudra: - extraire $900000 \mathrm{~m}^{3}$ de déblais rocheux, -w mettre en place $850000 \mathrm{~m}^{3}$ de remblais et $750000 \mathrm{~m}^{3}$ de béton,

-.. usiner, transporter et monter 30000 tonnes environ de matériel et d'appareillage.

Ces quantités donnent la mesure de l'ouvrage. Toutefois, si on les rapporte à la puissance disponible : $800000 \mathrm{~kW}$, comme à la production annuelle : 7 milliards de $k W h$, et en dépit des difficultés signalées, elles classent l'ensemble hydro-électrique de Sounda parmi les plus économiques et les plus rentables des aménagements réalisés ou projetés dans le monde à l'heure actuelle.

\section{DISCUSSION}

Président : M. Ligovzat

M. le Président remercie M. Fraxcov et prie les persomnes qui s'intéressent à la question de bien vouloir s'annoncer.

M. VArLet demande tout d'abord quel est le mode de calcul qui a été adopté pour le béton du barrage-voûte et qui a conduit à dire que l'on ne dépasserait pas un effort de compression de $60 \mathrm{~kg} / \mathrm{cm}^{2}$.

M. Fney (du Bureau d'lstudes Coyne et Bellier) explique que le calcul du barrage au stade d'avantprojet a été mené par les méthodes simples formule du tube et voutes actives) et sera complèté lors de l'étude définitive. Cependant, lordre de grandeur des contraintes annoncé par M. Fravcov, est dès maintenant, suffisamment approché.

M. Varfir a remarqué, d'autre part, sur le plan projeté, un col barré, c'est-à-dire qu'il y avait à cet endroit un barrage definitif que l'on n'utilisait pas comme evacuateur de crues, alor's qu'il y avait des évacuateurs de surface au barrage lui-meme; cette solution n'est pas toujours favorable et risque de conduire à un échec comme à Castelnau-Lassouts.

M. Francov confirme que c'est au-devant de risques analogues que l'on serait allé en adoptant une telle solution. Il précise que le déversement par l'ouvrage du col rive gauche a été envisagé, mais qu'il a fallu rapidement y renoncer : le thalwer auquel on s'adresserait a un bassin versant pratiquement nul; son débit naturel est insignifiant, et la qualite du terrain ne permet pas d'y envisager le déversement des crues du Kouilou, même compte tenu de l'amortissement que procurera la tranche supérieure de la retenue.

M. VARtes demande encore une précision; quelle est la puissance de l'alternateur?

M. Fratcov donne le chiffre de 242 MVA.

M. Fraxcoy ayant indiqué que les pivots de l'usine de Sounda supporteront une charge de $1400 t$, supérieure à celle admise dans les usines du Rhóne, M. Defatrre fait observer que les groupes de l'usine André Blondel ont précisément été définis de manière que la charge des pivots, y compris la poussée hydraulique maximum, soit de $1400 \mathrm{t}$ (contre $1600 \mathrm{t}$ à cette époque au Grand Coulee). Trois types différents de pivots sont utilisés a André Blondel, dont deux domnent satisfaction.

M. le l'ésident remercie M. Drsatrue de la confirmation qu'il apporte sur les dispositions posées pour le Kouilou.

M. Chamayou répond à la remarque de M. Delattre en précisant qu'en fait, les pivots mentionnés par M. Fravcou seront chargés à $1600 \mathrm{t}$, ce qui correspond bien a la surcharge de $10 \%$ vis-à-vis du Rhône, annoncée dans la communication.

D'autre part, les pivots maxima connus sont ceux de Bratsliaia, sur un affluent du Iénissèi, qui sont annoncés par les Russes comme pouvant supporter une chargo de $3000 \mathrm{t}$.

Mais, comme le fait remarquer M. Delatene, ces pivots ne sont pas construits.

M. de Maubranc intervient ensuite pour exprimer ses sentiments de reconnaissance à l'équipe d'E.D.F. qui a mené ces études avec tant de compétence et tant de succès. L'équipe de départ a dû certainement avoir à faire beaucoup de reconnaissances sous la chaleur dans la forêt tropicale, diffictile d'accès, et de ce fait, a eu beaucoup de mérite.

Quant au projet, la difficulté de la réalisation réside dans le fait qu'il s'agit d'une chute presque trop belle, en ce sens qu'elle se trouve seule dans un pays qui n'a pas encore l'électricité et à qui elle donne d'un seul coup 7 milliards de $k$ Wh à dépenser. En tout cas, l'isolement de cette très rrosse chute obligera à l'étudier de très près et a ne prendre aucun risque dans les dispositions techniques qu'il faudra finalement adopter, tant pour le génie civil que pour les groupes. Il n'est pas pensable, en effet, qu'après avoir mis en service un groupe capable de 1 milliard $1 / 2$ de $\mathrm{kWh}$ par an, avoir demarré l'industrie qui consommera cotte énergie, on puisse l'arrèter mème un jour.

M. le Président remercie M. de Mavblang de ses paroles flatteuses pour l'équipe d'E.D.F.

M. Aubers fait ensuite une remarque qui touche l'ensemble du projet, à propos de l'utilisation maximum d'un cours d'eau : 
* Par son ampleur mème, le projet, dont les grandes lignes viennent de nous etre exposées d'une maniere si claire, est de nature, en cas de réalisation, a réagir profondement sur l'économie de toute la région avoisinante. fes réactions doivent etre supputes non seulement poul fimmediat, mais aussi en envisageant des périodes plus. lointaines, au cours desquelles cette portion de l'Afrique. actuellement peu peuplée, deviendra peut etre le siege d'une activite beaucoup plus intense. Dans ces condilions, la question de l'utilisation, pour Ja navigalion, d'un lac de $270 \mathrm{~km}$ de long présenterait un gros intét. le coùt des transports élant, comme on le sait, un des principaux obstacles a la mise en raleur rapide des pays insulfisamment developpes.

\& Au surplus, l'idee progresse el kend it simpose partout dans le monde, gue lamenagement d'un cours d'eau a un point de rue rxclusif cqu'il s'agisse de la production d'energie, de la mavigution ou de fout autre objet) correspond it un reritable gaspillatge dont les conséquences peuvent stre tres fachouses. Il est vai que si fon met a part les deux brillantes exceptions fus constituent la Compagnie Nationale du lihone of lit sociéte allemande Rhin-Main-Danube, celle jdec parai! assez difficile a acclimater an Europe. Il ne faut pas en conclure qu'elle n'est pas adaptec at I'Arique.

\& Dans l'Jypothese où te lac de Sounda deriendrail le siège d'une navigation importante, la question du rac cordement a la mer devait exidemment se poser. Or, it se trouve que ce mème probleme a bé examine pour ho Konkouré al que le B.C.E.O.M. a etudie non seulement Ia navigation sur le bas-lionkource mais aussi le fran chissement par les bateaux de lat chute de Sonapili Cette etude a inontré qu'un exuloire de crues débon

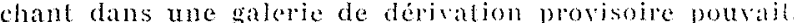
lorsqu'il s'agit d'un rocher de bonne qualite, moyennant un supplément de dépenses modeste, otre lansformó en un ouvage à deux fins. Celui-ei serait utilise peut-etre seulement deux ou trois fois par sièle comme exutoine dc crues, mais il servirail journellement comme ecluses. Si paradoxal que la chose puisse paratre au premier abord, une écluse de 100 metres environ de hauteur est parfaitement exploitable et rentable lorsque l'on dis pose d'un débil considéable el que, par conséquent, do prix du metre cubs deau consomme dans les éclusages est très faible.

\& La remarque qui precede me eonduit a demander s'il serait possible d'examiner dans quelle mesure on pourait envisager de concilier les exigenees de la production de l'énergie a Sounda avee l'intérêt qu'il y aurat a offrir des facilites à une navigation éventuchle.

Bien entendu, lexamen en question ne pourait etre anvisage que si l'nu dispose encore du temps nécessaire. du surplus, si l'opération dont je viens de parler etait reconnue possible et interessante, il faudrait cue les frais colrespondants puissent etre pris en charge par la Puissance publique. $\Rightarrow$

M. Te Président remercie M. Araknt de ses sugrestions al confirme que si le problème a été examiné par le lineau Central des Btudes de la France doutre-Mer pour le Konkoure, la question est tout a fait vierge en ce qui concerne le liouilou.

M. Javeons mppelle qu'atux vin Joumés de l'Hydrat lique de juin delnier, l'un des rapports présentés sur lincidence de l'evolution do la technologie des turbines sul le genic civil des centrales a montré, sous forme de courbes atablies pour chaque type de turbine, la variation du volume spécifigue du aste ciril d'une trat vec de soupe, a savoir le lapport $V / O$ en fonction du tébit unitaire du stoupe Q.

Il scrait interessant de voir comment se placerait, su ces graphiques, le point figuratil des groupes du Louilow, ctant donné gu'il s'agil de groupes constituant une large extrapolation par rapport aux réalisations en turbines Francis existantes, tant en France, qu'a l'etranger $\left(240 \mathrm{~m}^{3} / \mathrm{s}\right.$ a lieu de 120 a 150$)$ et d'en degager, le cas cehcant, l'incidence de la disposition en quinconec des groupes prérue sur le projet.

La meme question s'appliquerait d'ailleurs aux groupes de Soumiti dont a parlé précédemment II. Smos, compte tenu cependant du fait qu'il s'agit là d'une usine soutervine.

I. Fraxcor examinera la question.

H. le President remereie toutes les personnes gui ont pris la parole et dont les interventions ront donner lieu a de monvelles reflexions quant aux projets qui ont éte cxposes.

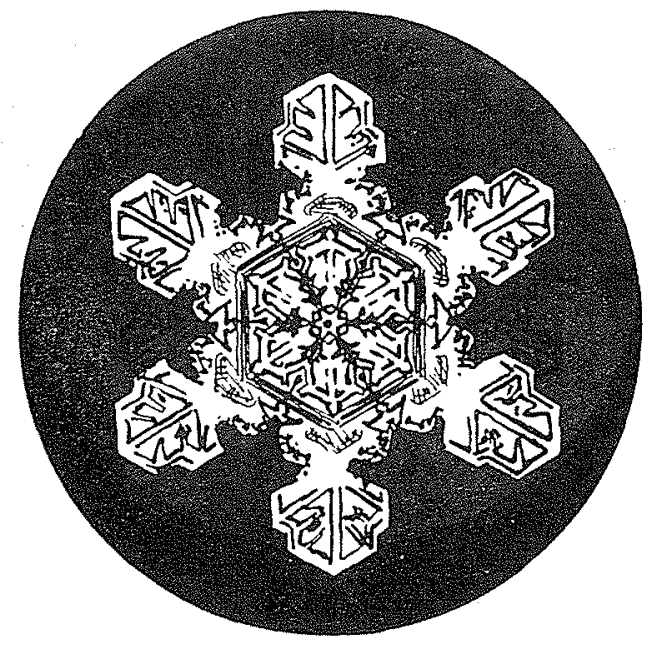

